\title{
Automatic Detection of Optical "Faults" in Communications Networks
}

\author{
——An Efficient, Fast, Physical Layer Monitoring Approach for Access Networks
}

\author{
Meir Bartur \\ Optical Zonu Corporation, Delano Street, Van Nuys, CA, USA \\ Email: meir@opticalzonu.com
}

Received 2013

\begin{abstract}
An efficient, fast, Physical Layer monitoring approach is needed to instantaneously identify, locate and report (via SNMP) optical fiber cuts, breaks or other faults (open or dirty optical connectors) in the optical fiber cable plant.
\end{abstract}

Keywords: Optical; Fiber; Fault; Monitoring; Physical; Layer; Single; Wavelength

\section{Introduction}

Today there is no proven method for automated monitoring of the optical fiber cable plant in the aggregation and data center segments of private campus or public communications networks. Metrics at the higher network layers may identify that a problem exists, but they cannot quickly isolate the location of an optical fiber fault nor can they automatically trigger the immediate dispatch of repair technicians.

An efficient, fast, physical layer monitoring approach is needed which can instantaneously identify, locate and report (via SNMP) any optical fiber cuts, breaks or other faults (as well as any open, damaged or dirty optical connectors) in the optical fiber cable plant. New technology is now available to accomplish this, which is backward compatible with legacy networks, and also forward compatible with new DWDM, 10G (and beyond) emerging networks, and may be easily integrated into SNMP monitoring of existing Switch/Router Equipment with minimal software/firmware upgrades.

\section{Micro-OTDR (uOTDR)}

\subsection{Structure of the Access Network}

The Aggregation and Data Center Segments of Private Campus or Public Communications Networks may be considered as the "edge" of the overall Access Network. Considering the breakdown of optical link distances in the Access Network [1], about $80 \%$ of the links consist of distances of $10 \mathrm{Km}$ or less, while virtually all of the Access Network links fall within the range of $40 \mathrm{Km}$ and below. While the data throughput of any particular Access link might not match that of its Long Haul "cousins", the fact that there are so many splices, connections, patch-panels and kilometers of optical fiber in this segment makes it particularly vulnerable to a host of optical "faults". Optical cables may be cut, optical connectors left open or become "dirty”, unexpected power outages may occur...a broad range of potential problems which are magnified by the sheer number of potential failure points.

\subsection{Fault Detection Must be Fast, Distributed and Pervasive}

To materially improve the reliability of the Access Network, fault detection must be fast, distributed and pervasive.

The detection of a fault needs to be fast so that the operation of the network, particularly an Ethernet-based one, is minimally impacted. A fault should be detected, located and reported to the Host Switch within a fraction of a second. If the fault is momentary, then the link should instantly recover. If not, then there ought to be the capability to initiate a series of diagnostic and/or repair options. Such responsiveness to a service disruption may translate directly into improved Service Level Agreements (SLAs) and the competitive advantages they may offer the Communications Service Provider (CSP).

Having a sophisticated piece of equipment monitoring a small number of optical fibers (thereby "consuming" these fibers, making them unavailable for revenue generation), is not very efficient in the extremely high link density environment found at the "edge" of the Access Network. We know that the link distances are relatively short (under $40 \mathrm{Km}$ ) and that the performance requirements of an OTDR to operate in this segment are modest. 
Perhaps a large number of moderate performance monitors, and their associated OTDRs, distributed throughout the Access Network, makes more sense than fewer, higher performance (and much more costly) systems.

In order to have the highest level of effectiveness, these distributed moderate performance monitors and OTDRs need to be virtually everywhere. Near 100\% fault detection may be achieved when large numbers of (or preferably all) optical links incorporate this performance monitoring. Fiber fault detection is based upon the reflected signal from the point of fault.

Fiber faults and intermittent connections present optical reflections of varying intensities. The reflection intensity of a fiber break has a known statistical distribu- tion. The distribution of the return signal from a fiber break/cut has been empirically determined and is presented in Figure 1.

As indicated in Fiber Fault Histogram of Figure 1 [2], to achieve a detection probability of greater than $95 \%$, the detection circuit must be better than $51 \mathrm{~dB}$.

Since the Fiber Fault Histogram indicates that the detection probability of an optical fiber fault follows a Normal Distribution, modeling to predict outcomes is straightforward. In fact, from the modest number of equations shown in Figure 2, a series of curves for the likely optical fiber cable deployments encountered in the Access portion of the Communications Network may be developed.

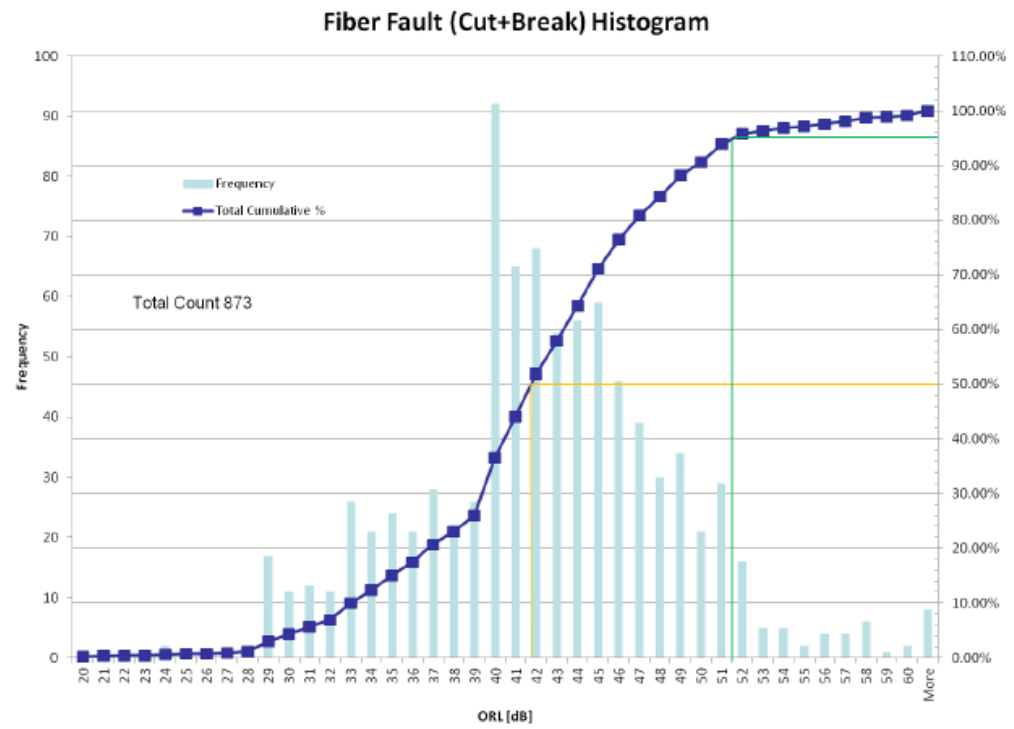

Figure 1. Fiber fault (Cut and Break) histogram.

$$
\begin{aligned}
& \alpha:=0.25 \frac{\mathrm{dB}}{\mathrm{km}} \text { Fiber attenuation } \\
& \mu:=42.3 \quad \sigma:=5.9 \quad \text { Measured, empirical data for normal distribution of fiber break ORL (dB) } \\
& \text { pnorm }(y, \mu, \sigma) \text { Returns the cumulative probability distribution for value } y \text {, for Normal distribution. } \\
& \mathrm{P}(\mathrm{S}, \mathrm{x}):=\operatorname{pnorm}(\mathrm{S}-2 \cdot \alpha \cdot \mathrm{x}, \mu, \sigma) \\
& \text { Single side detection - probability to detect a fault at a distance } x \text {. } \\
& S \text { is dynamic range (in } d B \text { ), } x \text { is distance. } \\
& \operatorname{PP}(\mathrm{S}, \mathrm{L}):=\int_{001}^{\mathrm{L}} \frac{\mathrm{P}(\mathrm{S}, \mathrm{x})}{\mathrm{L}} \mathrm{dx} \\
& \text { Single side detection - probability to detect any random fault } \\
& \text { of a link }(\mathrm{L} \mathrm{km}) \text {. } \\
& \operatorname{Pn}(\mathrm{S}, \mathrm{n}, \mathrm{L}):=\int_{.001}^{\mathrm{L}}\left[1-(1-\mathrm{P}(\mathrm{S}, \mathrm{x}))^{\mathrm{n}}\right] \mathrm{dx} \cdot \frac{100}{\mathrm{~L}} \quad \begin{array}{l}
\text { Single side detection - probability }(\%) \text { to detect any rand } \\
\text { fault of a link }(\mathrm{L} k \mathrm{~km}) \text { when more then } 1 \text { fiber strand is } \\
\text { monitored. }
\end{array} \\
& \operatorname{Pdn}(S, x, L, n):=2-(1-P(S, x))^{n}-(1-P(S, L-x))^{n}-\left[1-(1-P(S, x))^{n}\right] \cdot\left[1-(1-P(S, L-x))^{n}\right] \\
& \operatorname{PPd}(\mathrm{S}, \mathrm{L}, \mathrm{n}):=100 \cdot\left(\int_{.001}^{\mathrm{L}} \frac{\operatorname{Pdn}(\mathrm{S}, \mathrm{x}, \mathrm{L}, \mathrm{n})}{\mathrm{L}} \mathrm{dx}\right) \quad \begin{array}{l}
\text { monitored. } \\
\begin{array}{l}
\text { Both sides detection - probability }(\%) \text { to detect any rando } \\
\text { fault of a link }(\mathrm{L} \mathrm{km}) \text { when more then } 1 \text { fiber strand is } \\
\text { monitored. }
\end{array}
\end{array}
\end{aligned}
$$


Monitoring multiple fibers in the same cable trunk increases the detection probability (each fiber breaks differently). The optical fiber link may be monitored from one side, or from both sides, when a ring network topology is utilized. Monitoring both link ends, the detection probabilities of an optical fiber break which may be achieved are shown in the chart Figure 3.

Where is the most logical place to put this performance monitoring? It is in the optical transceivers, which by necessity, must reside at either side of every optical link in the Access Network. With performance monitoring distributed throughout the Access Network, essentially at both ends of every optical link, the Service Provider gains the highest probability of catching optical fiber faults in any portion of the Access Network. But is this approach feasible given the state of technology today?

\subsection{Single Fiber, Single Wavelength (SFSW) Transceivers}

In order for any transceiver to operate as an OTDR (as it must in order to fulfill the role of an optical fiber fault monitor), it must both transmit and receive at the same wavelength. The transceivers most widely deployed today are not of this type. However, recent advances in single fiber, single wavelength transceiver design, make the incorporation of OTDR functionality within the transceiver practical.

SFSW transceivers transmit and receive at the same wavelength (actually doubling the optical fiber plant capacity). Upon disruption of data link, or failure to connect, the transceivers could be designed to switch into a "Micro-OTDR" mode, emitting optical power pulses (> $+13 \mathrm{dBm}$ ) and detecting the reflected pulses at least down to $-42 \mathrm{dBm}$ optical power, for a dynamic range of at least $55 \mathrm{~dB}$, in order to detect reflections generated by physical faults in optical fiber link.

While not new, single fiber, single wavelength transceivers have in the past suffered from performance and operational issues related to their sensitivity to reflections...the exact thing which makes them work as OTDRs. Reflections within the optical subassembly of the transceiver itself, as well as reflections returning from the optical cable plant, all serve as noise contributors, adversely impacting optoelectronic performance. In certain situations, like an open Blue UPC (non-angled polish) connector, legacy single fiber, single wavelength transceivers were subject to "false locks" onto their own reflected signals. These concerns of the past have been resolved and robust single fiber, single wavelength transceivers are, in fact, available today.

Advancements in the design of single fiber, single wavelength optoelectronics, including near removal of internal optical crosstalk, allow these transceivers to more than make up for the optical budget loss penalties. New "smart" designs with microcontrollers add "state machine" firmware which makes the single fiber, single wavelength transceivers "immune" to reflections, for example from Blue UPC connector cable plants, while adding the functionality required for the transceiver to double as a micro-OTDR.

The additional functionality of an SFP transceiver with OTDR is depicted in Figure 4. Key aspects are the very high pulsed Laser Diode Driver which can generate 15 $\mathrm{dBm}$ peak pulse power, and the specialized threshold receiver that detects the reflections with higher sensitivity than the data receiver. Combined dynamic range of at least $55 \mathrm{~dB}$ is achievable.

\section{Fiber Fault Detection from at least one end}

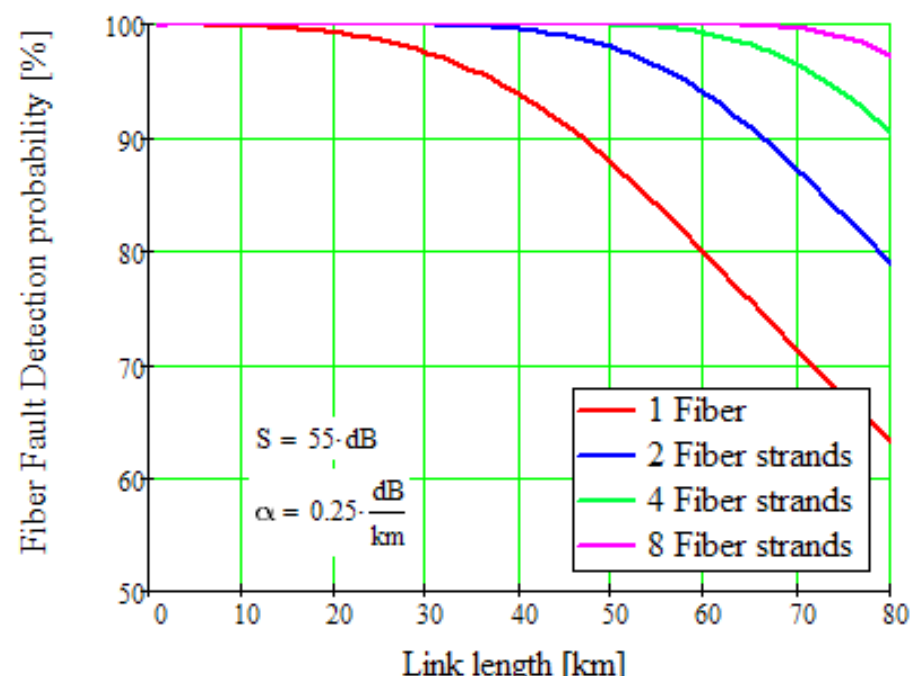

Figure 3. Probability of multi-strand detection depicted for 1, 2, 4 and 8 optical fibers monitored per bundle. 


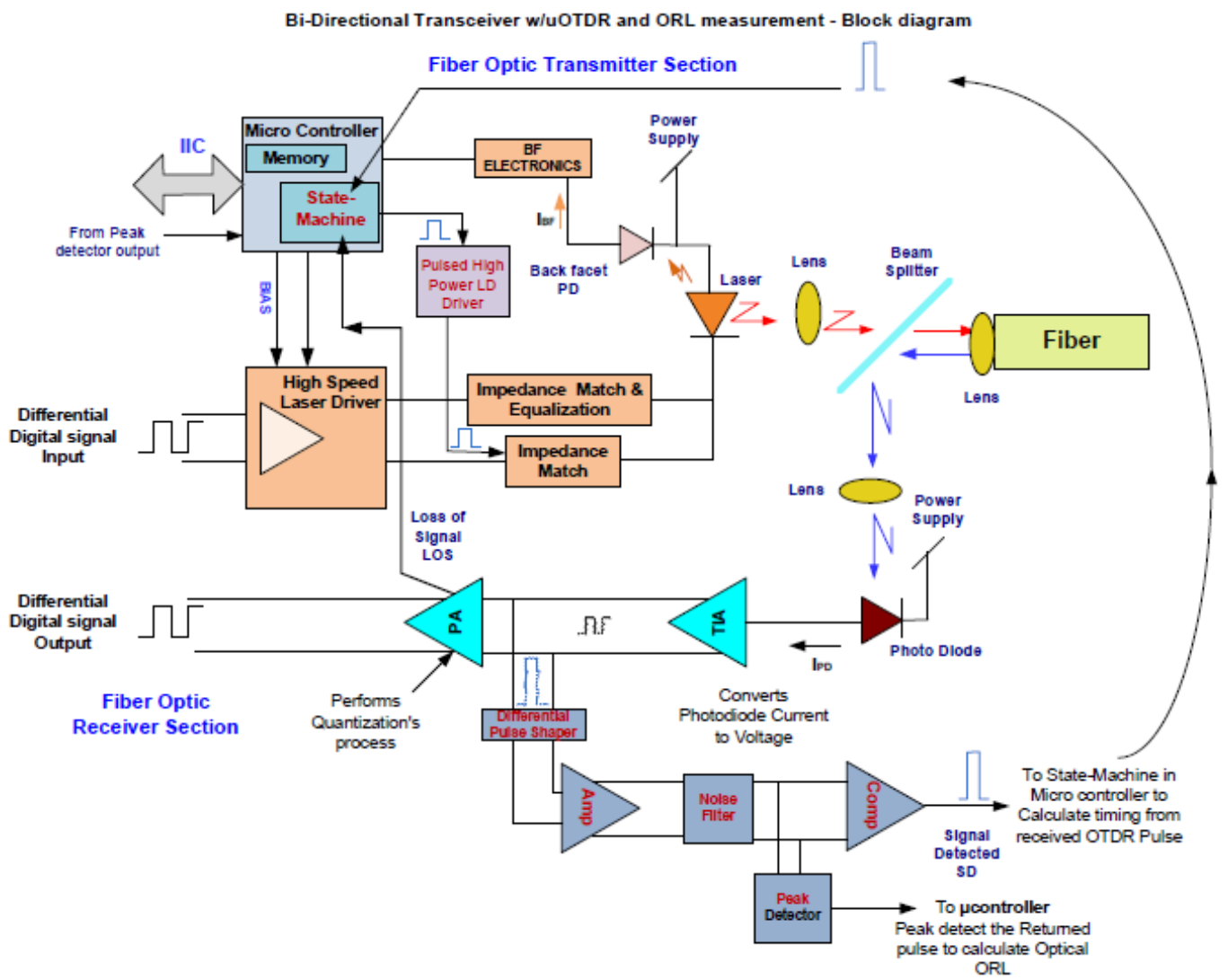

Figure 4. Functional block diagram of Micro-OTDR SFSW transceiver.

Table 1. Comparative benefits.

\begin{tabular}{lcl}
\hline \multicolumn{1}{c}{ Benefit } & Legacy & \multicolumn{1}{c}{ SFP+OTDR } \\
\hline Fast & No & Yes - Instantaneous. \\
Distributed & No & Yes - In the SFP. \\
Pervasive & No & Yes - At the end of every Link. \\
Cost & High & Low \\
Revenue & No & Yes - Carries Data. \\
\hline
\end{tabular}

\section{Conclusions}

There are available in the marketplace today, new technology single fiber, single wavelength transceivers which incorporate the functionality required to deploy fast, distributed and pervasive optical link monitoring with near $100 \%$ detection efficiency. These transceivers do not require specialty equipment but may reside in standard Host Switches which may be managed through industry standard SNMP systems, offering many benefits over legacy Remote Fiber Testing Systems.

The new single fiber, single wavelength transceivers do not suffer from any of the performance or operational drawbacks which plagued these designs in the past, and with their new capabilities provide the basis for robust automatic detection, localization and reporting of optical fiber cable plant "faults" in the Aggregation and Data Center Segments of Private Campus or Public Communications Networks.

\section{REFERENCES}

[1] Michael J. Hartmann, Unpublished Working Papers: Compendium of Multiple Industry Sources and Trade Press References. (2007-2012).

[2] Meir Bartur, Unpublished Working Papers: Summary of Empirical Data from Optical Fiber Cut Experiments. (2012). 